\title{
The Enigma of Cellular Immunity in Glomerulonephritis: Lupus Nephritis Is Not Necessarily a Prototype of Immune Complex-Mediated Glomerulonephritis
}

\author{
Edmund J. Lewis \\ Division of Nephrology, Rush University Medical Center, Chicago, III., USA
}

Kline Bolton has made important contributions to the field of nephrology, not the least of which is his work on T-cell immunity in glomerulonephritis. I would like to take you back into the history of that subject. It is a history that few people know.

In 1964, when I was a resident at the Johns Hopkins Hospital, I had the rare good fortune of participating in the care of Dr. Arnold Rich, who was the retired chairman of the Department of Pathology and one of the world's finest experimental pathologists. That year Lyndon Johnson was running for president against Barry Goldwater and Dr. Rich was a Goldwater supporter probably the only one in the entire city of Baltimore - and there wasn't much for us to talk about, because in those days I was actually a Democrat. Therefore, we talked about medicine. Dr. Rich was, for many years, the most prominent researcher in what we now call experimental immunologic diseases. Of course, his work was before antibiotics, so the way you treated pneumonia in those days was to inject a horse with pneumococci in order to get antibodies and you used horse serum to treat the patient. Some patients got serum sickness. Dr. Rich went into great detail in terms of the mechanism of serum sickness and how it could be related to other diseases in man. One day, after he was discharged, he sent me his Harvey Lecture from 1947 and it was about the pathogenesis of rheumatic fever, lupus, rheumatoid arthritis, and peri- arteritis, and how these diseases related to his work with serum sickness. It was a remarkable review of a life's work [1].

I went to Boston to Dr. Frank Austen's lab for my research fellowship and one of the people who was working there was Dr. John David. In the mid-1960s, when I was a fellow, experimental immune diseases of the glomerulus had been studied rather well, particularly by Dr. Frank Dixon, who was at Scripps. The important pathogenetic issue regarding glomerular diseases was the immunologic activity that caused inflammation in the kidney. Two mechanisms were accepted. One was immune complex disease associated with circulating immune complexes, as occurred in serum sickness. In lupus patients there is glomerular capillary inflammation, which is attributed to deposited immune complexes. Presumably, antibodies to DNA and circulating DNA polynucleotides form a complex, which leads to their deposition and glomerular inflammation. The second mechanism was autoimmune glomerulonephritis - which Kline has spent his career studying so beautifully. The classic example of glomerulonephritis associated with antibodies to glomerular basement membrane (GBM) is Goodpasture's syndrome. For reasons that are largely unclear, the body makes antibodies against an autoantigen in the GBM. Now I became quite involved with Dr. Rich and saw him during his multiple hospitalizations. Dr. Rich had published a

\section{KARGER}

Fax +4161306 1234 E-Mail karger@karger.ch www.karger.com

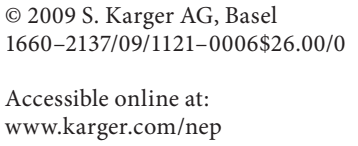




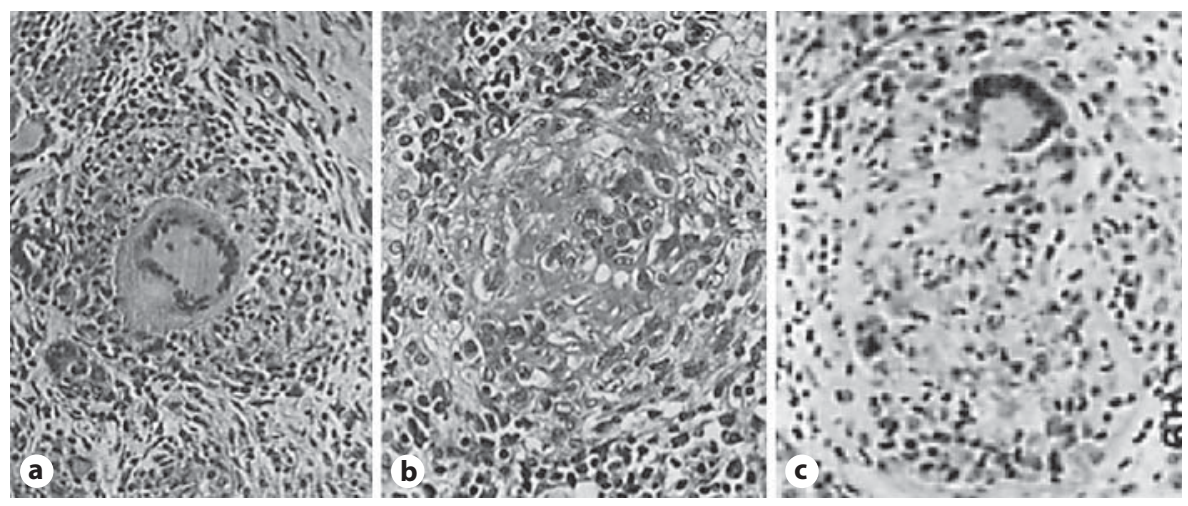

Fig. 1. Destructive inflammatory glomerular lesion appears to be similar to classic tuberculous granuloma. a A classic tuberculous granuloma from a case of tuberculous salpingitis. Note the multinucleated giant cell, characteristic of this granulomatous lesion. b A totally destroyed glomerulus from a patient who had antiglomerular basement membrane antibody-mediated glomerulonephritis. Note the mononuclear cell infiltrate, which has complete- ly replaced the collapsed glomeruli, and the surrounding lymphocytic infiltrate. c Another destroyed glomerulus from a patient with antiglomerular basement membrane-associated glomerulonephritis. The destroyed glomerulus appears as though it is a 'pseudo-granuloma'. Note the multinucleated giant cell present in the glomerulus. book that was well over a thousand pages long on the pathogenesis of tuberculosis [2]. If one were interested in tuberculosis today, one could read that book, and it would be pretty up to date in terms of many aspects of pathogenesis. The first edition was published in 1940 and Dr. Rich had written a remarkable description about immune system activity that occurs when someone gets tuberculosis. This is quite different from humoral immunity, because this involves the cells of the immune system becoming sensitized to the chemical composition of the tubercle bacillus; and it is not humoral antibody, but rather cellular immunity, that gets involved in trying to destroy these bacteria. A lot of the work cited in this 1,000-page volume was original work by Dr. Rich. For example, figure 1a is a granuloma made up of cells of the immune system which are there in order to attack tubercle bacilli. We now know that many of these cells are sensitized $\mathrm{T}$ lymphocytes and that they draw monocytes into the lesion, and these are able to engulf and destroy the bacteria. As noted in figure 1a, there are times when monocytes in these granulomas will actually fuse to form multinucleated giant cells.

Now, early in nephrology, I was young, and everybody who was seriously interested in nephrology was interested in renal physiology. Most of those who were interested in renal immunology were considered not smart enough to study physiology. One of the things that struck me as we examined more and more renal biopsies was that there were times when you saw a renal biopsy whose glomeru- li were completely involved with a destructive inflammatory lesion (fig. 1b). The capillaries could be completely destroyed and the cellular reaction, to my eye, looked a little bit like a tuberculous granuloma (fig. 1c). So I drew the conclusion that perhaps some of what was going on in these glomeruli was not just humoral inflammatory damage by antibodies and immune complexes, and that cellular immunity could somehow be involved. In some glomeruli one actually could see multinucleated giant cells. I went back to Dr. Rich's book, and one of the experiments that Dr. Rich reported became highly relevant [2]. He would take a guinea pig, and extract the peripheral white cells, which we now know represent $T$ cells and monocytes. Then he would take these white cells from normal guinea pigs and place them on a piece of glass and they would migrate along the slide. However, if the media in which the cells were placed contained the antigen tuberculin, the cells from normal animals would migrate normally, whereas in animals sensitized to tubercle bacilli, the cells would not migrate (fig. 2). When he looked at these latter cells he confirmed that it wasn't because they had died. They had somehow been inhibited from migrating. He proposed that there had been a migration inhibitory factor produced by these cells when they contacted an antigen to which they were sensitive. He concluded that this lack of migration away from an antigen such as tubercle bacilli allowed them to stay in the area in order to attempt to kill the bacteria. He did this experiment in 1932. 
Fig. 2. a A deposit of mononuclear cells, which have been placed on an agar-coated slide. Note the free migration of cells out of this deposit and across the surface of the plate. $\mathbf{b}$ A similar group of cells taken from an experimental animal which was sensitized to tubercle bacilli. The agar medium coating the slide contained tuberculin antigen. Note the lack of migration of monocytes away from this deposit of white cells, which Dr. Rich interpreted as being the result of a 'migration inhibitory factor' released by these cells (reproduced from Rich AR: The Pathogenesis of Tuberculosis. Springfield, Thomas, 1951, p 412).

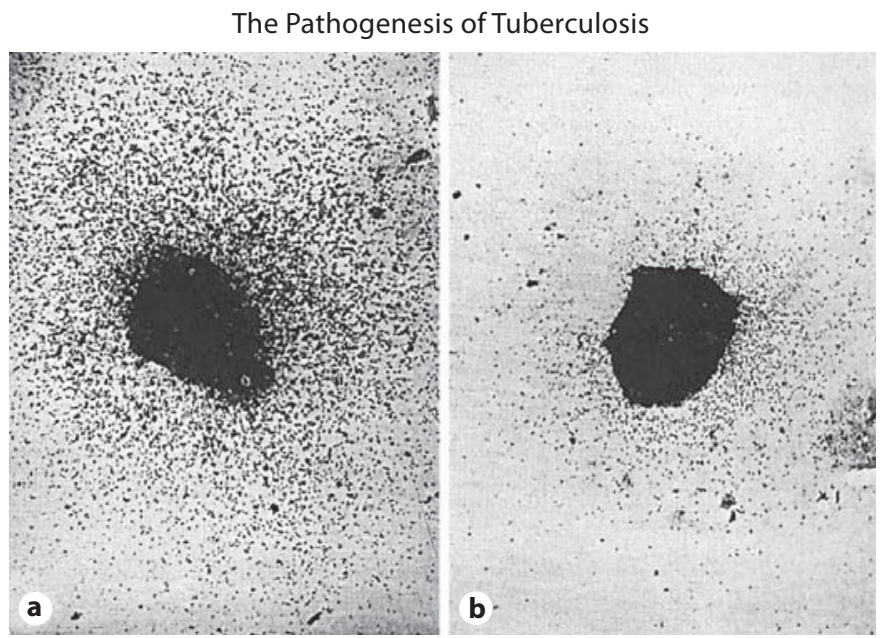

Fig. 3. Human peripheral monocytes have been placed in capillary tubes and then in culture medium. Note that when the cells were grown in medium that did not contain solubilized glomerular basement antigen (patient MG, left) there was migration out of the capillary tube. On the right the cells were incubated in medium, which contained solubilized human glomerular basement membrane from patient MG; note the lack of migration out of the capillary tube. This patient had antiglomerular basement membrane-mediated glomerulonephritis. In the lower portion of the figure (patient JM), the cells migrated out of the capillary tube, both under control conditions and when glomerular basement membrane antigen was in the medium. This patient had glomerulonephritis which was not associated with antiglomerular basement membrane antibodies.

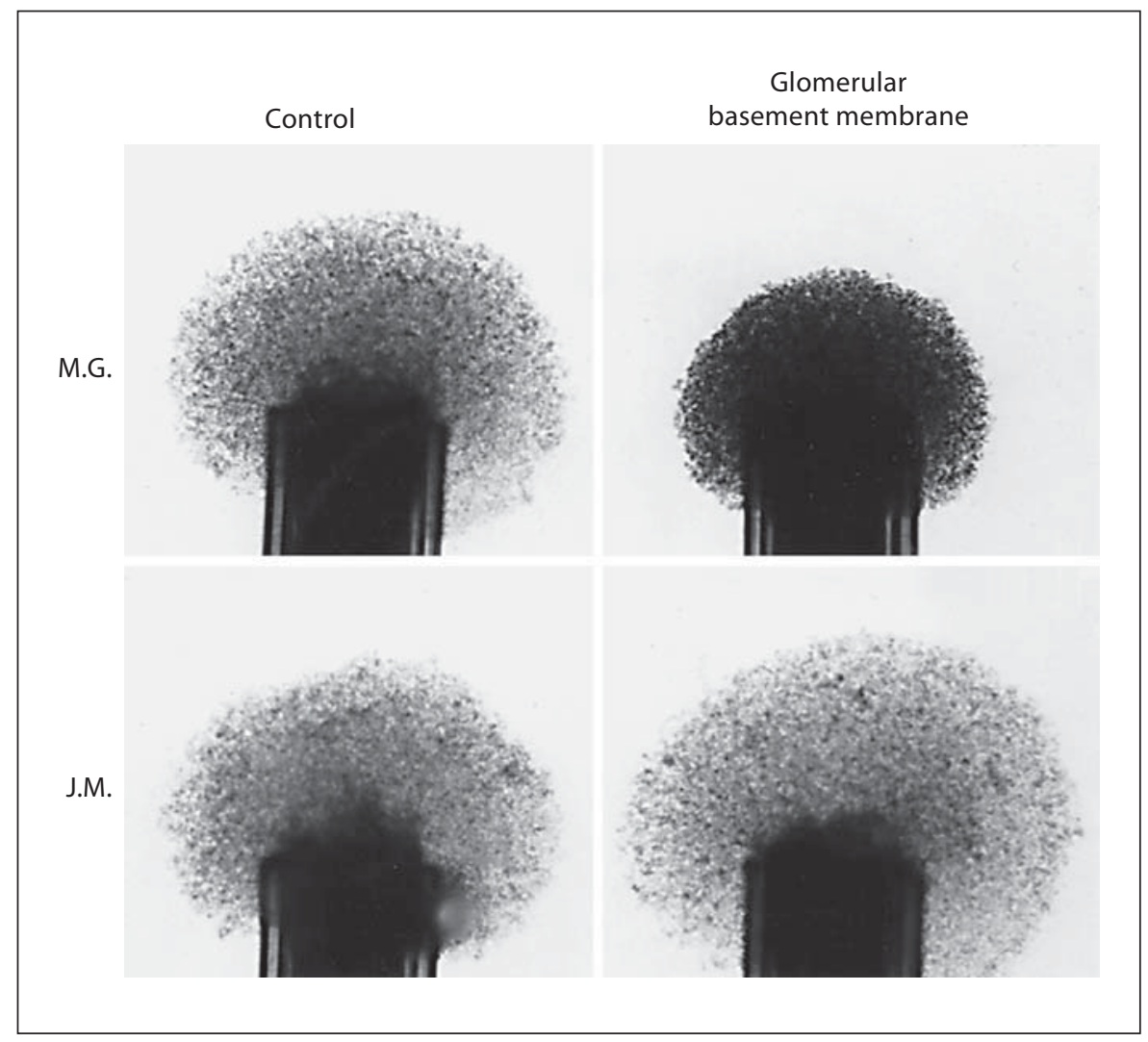

John David and his trainee, Dr. Ross Rocklin, wanted to quantify this phenomenon and so they took monocytes and T cells out of blood and put them in glass capillary tubes, and incubated them for $48 \mathrm{~h}$. Under normal circumstances you could see these cells migrating out of the tube. If you just incubated the cells in normal culture media, the cells would migrate out of the tube, and then one could actually quantify the surface area in order to see how much migration went on. David reproduced Dr. Rich's experiment and showed that cells from animals or 
humans that were sensitized to tuberculin would not migrate out of the capillary tubes if tuberculin was in the culture medium [3].

Because of the observation from some renal biopsies that some glomeruli in anti-GBM-associated glomerulonephritis actually looked like tuberculous granulomas, I was interested in studying such patients using this technique. What we found was that if you put solubilized GBM antigen into the culture medium, there were patients with anti-GBM disease whose cells did not migrate (fig. 3). My conclusion was that, just as Dr. Rich had shown with tuberculin, these cells indicated cellular hypersensitivity to GBM [4]. For controls, we looked at normals, and we looked at patients who had other renal diseases such as polycystic renal disease or patients who had glomerulonephritis not due to anti-GBM antibodies. Cells from these patients migrated normally. As far as I was concerned, we were showing cellular hypersensitivity to GBM in patients with anti-GBM-mediated glomerulonephritis and we published this in the New England Journal of Medicine in 1970 [4]. I concluded that these results indicated that in human glomerulonephritis there was a suggestion that sensitized lymphocytes may be involved in the pathogenesis of renal disease, which could explain the granulomatous nature of destroyed glomeruli that we observed in these patients. This article was accompanied by an editorial by the most important man in renal immunology, Dr. Frank Dixon, who wrote the most malicious editorial that I, to this day, have ever read [5]. It basically said that the results were artifactual and that I was nuts. At this point, Bill and Kline, who were residents on the Harvard Service at Boston City Hospital, walked into my office and, for whatever reason, agreed to work with me. That was the beginning of things, and I have to give Kline all the credit in the world, as he has been so effective in teasing out this subject, an important area of immune pathophysiology of glomerular diseases. Kline persisted in his quest of the significance of sensitized T cells in glomerulonephritis and, in the end, was remarkably successful.

In 1971 Kline, Bill Couser and myself moved to the University of Chicago. Our experience at the University of Chicago was complex and it was not clear if we could pursue our interests as we wished. My predecessor at Rush was Bob Kark. Dr. Kark had developed the technique of percutaneous renal biopsy, which established the field of renal pathology and allowed him to describe diseases such as lupus nephritis in detail. He had trained at the Harvard Service at the Boston City Hospital and we had become quite good friends before we moved to Chi- cago. When Bob decided that he was going to retire, he called me wondering if I wanted his job at Rush. I didn't really enjoy the University of Chicago, and so it was like a call from heaven. That was really what broke us up. It is a shame really, because if you can imagine that had Kline, Bill and myself stayed at the University of Chicago, there would have been a lot of pretty good renal immunology that would have been done at that institution. Anyway, we split: Kline came here to the University of Virginia, Bill returned to Boston to Boston University, and I went to Rush. One of the reasons that I took the job was that Bob Kark had developed renal biopsy and so he had an enormous experience in lupus with a large traditional referral pattern. Ironically, lupus nephritis is the prototypical immune complex disease. There are circulating antibodies to an antigen, and you can show circulating and deposited immune complexes by a variety of mechanisms. The evidence that lupus nephritis was an immune complex-mediated disease was reported more than 35 years ago.

It was believed that glomerular inflammation resulted from deposited immune complexes, which formed aggregates in the glomerular capillaries and therefore were causing the inflammation. And you can readily show evidence of immune deposits by immunofluorescence microscopy. The concept that grew was that there was a continuum: the more of these complexes, the more inflammation in the glomeruli. Oddly enough, we published in 1983 that we had 4 cases of lupus where we couldn't find any complexes and that the lesions were akin to those seen in the pauci-immune glomerulonephritis of microscopic polyarteritis, a lesion described by Kline and Bill. We wondered whether the segmental glomerular lesion and the diffuse lesion were not simply quantitatively different depending upon the amount of immune complexes deposited, but were actually qualitatively different lesions pathogenetically.

A number of years ago we did a study of plasmapheresis in patients with severe lupus nephritis, where you take the plasma out of the patient and replace it with normal plasma, thus removing from the blood preformed immune complexes, free antibodies and, presumably, antigens [6]. Many people thought that this treatment would cure lupus nephritis, but it didn't. The immune complex levels came down, the antibody levels came down, the abnormal humoral immunological tests seen in lupus nephritis started to look normal very quickly, but the kidney disease really did not do that well. One of the things that we found was that the patients who had segmental glomerular lesions actually did much worse than the pa- 
tients who had diffuse glomerular lesions, and that doesn't follow if the continuum of quantity of deposited immune complexes being related to injury holds true [7]. So we wondered about why that might be the case. And when we looked at the pathology, we found that in the diffuse glomerulonephritis the abnormalities were compatible with immune complex disease, and that these patients responded to standard immunosuppressive and anti-inflammatory therapy. However, in the segmental glomerulonephritis a different picture arose. The abnormalities were more compatible with glomerular necrosis seen in microscopic polyarteritis (i.e., pauci-immune glomerulonephritis) [8]. So we started to look at our data, which revealed that in segmental glomerulonephritis, if you look with electron microscopy for subendothelial deposits, which is the classic humoral-mediated abnormality of lupus, you often find no deposits or a very small amount, and rarely do you see the massive deposits of immune complexes in glomerular capillaries which are characteristic of diffuse proliferative glomerulonephritis. So we have concluded that most of the patients with segmental lesions really didn't have much in the way of pathology due to humoral immunity. On immunofluorescence microscopy you see the same picture. Thirty percent of patients with segmental glomerulonephritis have no IgG in the peripheral capillary loops, some have a very small trace, and some have 1+; so from our point of view, there appear to be two different things going on. The segmental lesion and the diffuse lesion are histologically, immunologically, ultrastructurally distinct lesions, and the seg- mental lesion is not the prototypic humoral lesion described in lupus nephritis. The clinical outcomes in the segmental glomerulonephritis are different, which leads one to treat these patients much more actively. Just as the original work regarding cellular hypersensitivity was greeted with doubt, so too was the concept that lupus nephritis is not simply an antigen-antibody disease but may even involve cellular hypersensitivity. And so I quote Homer Smith, who was the father of renal physiology: 'We must view with profound respect the infinite capacity of the human brain to resist the introduction of useful knowledge' [9]. Certainly this is what happened when the subject of cellular immunity in glomerulonephritis was raised, and surely Kline is well positioned to support this unfortunate truth. My message to young investigators is that you have to find a problem that should be a big problem, just as Kline did. It must be a big problem, so that as exemplified by Kline, the amount of time and dedication that you must invest is worth it. Your results may not be what the establishment expects or accepts. Just remember, just as Kline did, about the infinite capacity of the human brain to resist the introduction of useful knowledge. He stuck with it, and he showed that there was indeed a non-humoral pathogenesis going on in glomerulonephritis and, Kline, I congratulate you on that and I think that the subject has now come full circle as we are learning more and more about lupus. As the result of Kline's remarkable imaginative work we can now look at glomerulonephritis with new eyes. And that is the nature and the price of progress.

\section{References}

1 Rich AR: Hypersensitivity in disease with especial reference to polyarteritis nodosa, rheumatic fever, disseminated lupus erythematosus and rheumatoid arthritis. Harvey Lecture Series 1946-1947;42:106-147.

2 Rich AR: The Pathogenesis of Tuberculosis. Springfield, Thomas, 1951, p 412.

3 David JR, Al-Askari S, Lawrence HS: Delayed hypersensitivity in vitro: I. The specificity of inhibition of cell migration by antigens. J Immunol 1964;93:264-273.
4 Rocklin RE, Lewis EJ, David JR: In vitro evidence for cellular hypersensitivity to glomerular basement membrane antigens in human glomerulonephritis. N Engl J Med 1970;283:497-501.

5 Dixon FJ: What are sensitized cells doing in glomerulonephritis? N Engl J Med 1970;283: 536-537.

6 Lewis EJ, Hunsicker LG, Lan SP, Rohde R, Lachin J; The Lupus Nephritis Study Group: A controlled trial of plasmapheresis therapy in severe lupus nephritis. N Engl J Med 1992; 326:1373-1379.
7 Najafi C, Korbet SM, Lewis EJ, Schwartz MM, Reichlin M, Evans J; Collaborative Study Group: The significance of histologic patterns of glomerular injury upon longterm prognosis in severe glomerulonephritis. Kidney Int 2001;59:2156-2163.

8 Lewis EJ, Schwartz MM, Korbet SM: Severe lupus nephritis: importance of re-evaluating the histologic classification and the approach to patient care. J Nephrol 2001;14:223-227.

9 Smith HW: From Fish to Philosopher. Boston, Little, Brown, 1953, p 184. 